Revue d'histoire de l'Amérique française

REYUE D.HISTOIRE DE L'AMÉRIQUE FRANÇAISE

\title{
Civilisation : terminologie et structure
}

\section{Roland Lamontagne}

Volume 18, numéro 3, décembre 1964

URI : https://id.erudit.org/iderudit/302388ar

DOI : https://doi.org/10.7202/302388ar

Aller au sommaire du numéro

Éditeur(s)

Institut d'histoire de l'Amérique française

ISSN

0035-2357 (imprimé)

1492-1383 (numérique)

Découvrir la revue

Citer cet article

Lamontagne, R. (1964). Civilisation : terminologie et structure. Revue d'histoire de l'Amérique française, 18(3), 357-362. https://doi.org/10.7202/302388ar d'utilisation que vous pouvez consulter en ligne.

https://apropos.erudit.org/fr/usagers/politique-dutilisation/ 


\section{CIVILISATION : TERMINOLOGIE ET STRUCTURE *}

Attentive aux problèmes contemporains, chaque génération recompose son histoire en vue d'interpréter le passé à la lumière de nouvelles recherches sur la condition humaine. A la façon de l'individu qui reprend son passé, immédiat ou éloigné, c'està-dire selon un rythme rapide ou lent (pour employer une expression de Fernand Braudel), ainsi en est-il du groupe social. Dans une perspective d'interprétation historique étendue à l'ensemble de l'activité humaine, compte tenu de la durée relative aux individus et aux sociétés, il convient d'acheminer à l'unité d'une pensée cohérente, en fonction de la structure des civilisations, la multiplicité et la variété du réel tel que transmis par nos sources d'archives.

L'enquête historique révèle les valeurs de progrès, les axes de développement et les facteurs de ralentissement ou d'inertie. Quelques exemples illustrent ce double aspect de progrès et d'inertie au même temps historique. La France, dans la première moitié du XVIII ${ }^{\mathrm{e}}$ siècle, exporte ses biens culturels à travers l'Europe; elle néglige l'augmentation des colonies. Un mouvement réformiste se précise avec Vauban, Maurepas, La Galissonière, Machault, Silhouette, Turgot; la classe politique s'y oppose vivement. Les disgrâces de Vauban, Maurepas, Turgot manifestent une action brimée.

* Monsieur le $\mathrm{P}^{r}$ F. Furet de l'Ecole Pratique des Hautes Etudes, (VI ${ }^{\mathrm{e}}$ Section) Sciences Economiques et Sociales, a eu l'obligeance de me communiquer de précieuses indications pour ce travail, dans ses lettres en dates du 15 octobre 1963 et du 3 juin 1964 . 


\section{TERMINOLOGIE}

La définition $d u$ vocable de civilisation présente des difficultés. ${ }^{1}$ Les écrits des spécialistes de sciences humaines renferment de multiples variations terminologiques. Le mot de civilisation remonte au XVIII ${ }^{\text {e }}$ siècle français. Il se rattache à l'adjectif "cultivé" qui signifiait: avoir de bonnes manières. Le substantif police, qui découle de "poli", synonyme de "civilisé", figure dans les documents d'histoire de la Nouvelle-France: il désigne l'administration interne.

Au milieu du XVIII ${ }^{e}$ siècle, Turgot emploie le mot de civilisation dans le sens de "passage à l'état civilisé". Par opposition aux peuples primitifs, la civilisation, à la fin du règne de Louis $\mathrm{XV}$, reflète la société française. Ce terme se chargeant d'un contenu nouveau commence son voyage à travers l'Europe. Dès le premier quart du XIX $\mathrm{X}^{\mathrm{e}}$ siècle, une ambiguïté recouvre le vocable de civilisation qui équivaut à culture, chez les auteurs allemands. Au $\mathrm{XX}^{\mathrm{e}}$ siècle, des pays de l'Europe orientale suivront l'exemple de l'Allemagne qui accorde la préséance au terme de culture, au détriment du mot de civilisation qui a tendance à désigner l'organisation industrielle d'une société. Sur la lancée des ouvrages d'anthropologie, le terme de culture s'applique aux sociétés nomades ou semi-nomades. En France, en Grande-Bretagne et en Amérique du Nord, le mot civilisation représente l'ensemble des facteurs de vie collective d'une ethnie. Il englobe également l'aspect culturel qui se situe sur le plan des idées et des diverses formes de l'art. Face à ces multiples complications terminologiques touchant les civilisations, se dresse une civilisation avec ses effets de planification et de transformation à l'échelle planétaire.

\section{STRUCTURE}

Le peuplement est la condition fondamentale de la formation d'une civilisation. Bougainville a saisi l'importance de la densité de peuplement. ${ }^{2}$ Dans une étude de l'hypothèse relative à l'éven-

${ }^{1}$ Voir l'ouvrage de S. Baille, F. Braudel et R. Philippe, Le Monde Actuel. Histoire et Civilisations (Paris, 1963).

2 Densité de peuplement $=\frac{\text { Nombre d'occupants }}{\text { Surface de terre habitée. }}$ 
tualité d'un échec militaire suivi d'un traité qui accorderait à la France une partie de son ancien territoire, il écrit: "nous ne regretterons pas que les limites en soient resserrées; même perdant une étendue considérable de terrain, nous ferons un profit qui ne sauroit se calculer. Le Canada ne sera plus ce vaste corps étique dont les branches projetées à une distance infinie de leur tige tenoient à peine au tronc et l'affamoient (...) La France possédera une colonie d'une étendue raisonnable dont les frontières rapprochées du Centre seront susceptibles d'une défense et meilleure et plus facile. Cette colonie rétrécie et plus nerveuse se peuplera à vue d'œil..." ${ }^{3}$ Bougainville poursuit son analyse; il explique qu'en fonction de la densité de population, une société peut atteindre un niveau de vibration ou de dynamisme propice à la diversification des moyens d'action et constituerait ainsi un pôle d'attraction des immigrants.

Compte tenu de la lenteur de formation des civilisations, les apports successifs des générations, au sein d'une même ethnie, dans des attitudes d'accueil ou d'opposition vis-à-vis des influences extérieures, modifient le cheminement d'une société, à l'intérieur de certaines limites de continuité. L'interdépendance des facteurs de civilisation d'ordre religieux, politique, économico-social, technique, culturel, psychologique, est intimement liée à la vie d'une société dans un milieu géographique.

En élargissant le champ d'exploration de la Nouvelle-France, les recherches devraient tenir compte de la théorie d'ensemble d'interprétation historique. ${ }^{4}$

ROLAND LAMONTAGNE

Université de Montréal.

${ }^{3}$ Archives du Séminaire de Québec, Mss. 19, fos 5-6. Le mémoire de Bougainville, "Considérations sur l'état présent du Canada", figure également sous la même cote; il est un complément au mémoire intitulé "Considérations sur le Canada", d'où est tirée la citation que nous venons de lire. 4 John U. Nef, Les Fondements culturels de la civilisation industrielle (Paris, 1964). - L'ouvrage de Fernand Braudel, La Méditerranée et le Monde méditerranéen à l'époque de Philippe II (Paris, 1949), constitue une des plus brillantes synthèses à l'appui du principe d'interprétation historique de mouvement d'ensemble. 


\section{A N N E X E}

TABLEAUX I et II

A partir d'un lieu d'observation, en l'occurrence la NouvelleFrance, il convient d'illustrer un aspect de structure de civilisation. Nous pourrions marquer sur les tableaux I et II un nombre indéterminé de faits historiques et de lieux géographiques, comme points de repère au temps présent dans un mouvement de durée à l'échelle humaine. Il s'agit d'éviter un durcissement de direction de recherche. Les facteurs de civilisation n'exercent pas leurs effets isolément, mais sous l'aspect d'une interaction.

Un schéma d'interprétation historique de quelques données démographiques et géographiques révèle des composantes sous forme de variables influençantes et influencées et de résultantes. La loi statistique des grands nombres n'infirme pas la liberté de vouloir des individus.

\section{Note explicative du graphique des coordonnées civilisation - milieu géographique}

a La civilisation est la projection de la vie d'une société. "Pas de civilisations sans sociétés qui les portent, les animent de leurs tensions, de leur progrès". ${ }^{1}$

b Les chances se présentent sous l'aspect des dons de la nature (v.g. climat, richesse des matières premières) et des moyens appropriés à l'utilisation de ces ressources, par opposition aux inconvénients tels que pauvreté du sol, sous-développement économique, etc.

c La notion de frontière varie. Ainsi l'Océan ne se présente plus comme une barrière, mais comme un moyen de communication.

d A travers les idées, les arts, les institutions et le mode d'organisation matérielle, des traits distinctifs se transmettent, en dépit des influences subies.

${ }^{1}$ Le lecteur devrait étudier avec soin l'étude de M. le $\mathrm{Pr}$ Fernand Braudel, sous le titre de grammaire des civilisations. Voir S. Baille, F. Braudel, R. Philippe, Le Monde actuel. Histoire et civilisations (Paris, $1963), 145-170$. D'après la note qui figure en page 7 , le livre II a été écrit par F. Braudel. 


\section{GRAPHIQUES DES COORDONNÉES}

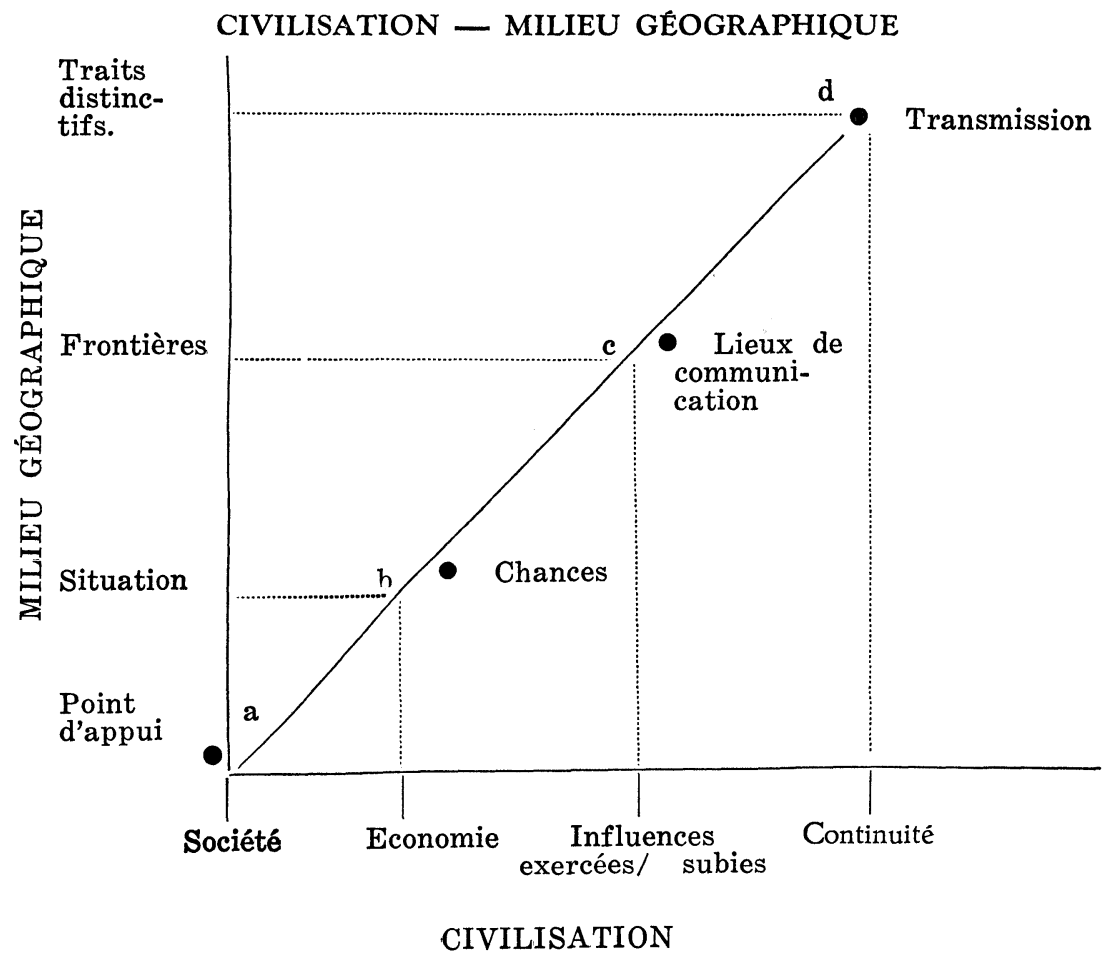


TABLEAU I

Interprétation historique de quelques données démographiques en fonction des variables influençantes, influencées et des résultantes.

\begin{tabular}{|c|c|c|c|c|c|c|c|c|}
\hline Variables & \multicolumn{2}{|c|}{$\begin{array}{l}\text { Mouvements } \\
\text { migratoires }\end{array}$} & \multicolumn{2}{|c|}{ Economie } & \multicolumn{2}{|c|}{ Militaire } & \multicolumn{2}{|c|}{ Politique } \\
\hline $\begin{array}{l}\text { Influan- } \\
\text { çantes } \\
\text { encées }\end{array}$ & $\begin{array}{l}\text { Immi- } \\
\text { gration }\end{array}$ & $\begin{array}{l}\text { Emi- } \\
\text { gration }\end{array}$ & $\begin{array}{l}\text { Mode } \\
\text { de vie } \\
\text { rurale }\end{array}$ & $\begin{array}{l}\text { Enrichis- } \\
\text { sement }\end{array}$ & Guerre & $\begin{array}{l}\text { Transfert } \\
\text { de popu- } \\
\text { lation }\end{array}$ & $\begin{array}{c}\text { Décision } \\
\text { de } \\
\text { Louis } \\
\text { XIV }\end{array}$ & $\begin{array}{l}\text { Politique } \\
\text { popula- } \\
\text { tionniste }\end{array}$ \\
\hline $\begin{array}{l}\text { Compo- } \\
\text { sition } \\
\text { démo- } \\
\text { graphique }\end{array}$ & $\begin{array}{l}\text { Accrois- } \\
\text { sement } \\
\text { de } \\
\text { jeunesse } \\
\text { relative }\end{array}$ & $\begin{array}{c}\text { Facteur } \\
\text { de } \\
\text { vieillis- } \\
\text { sement } \\
\text { relatif }\end{array}$ & $\begin{array}{l}\text { Augmen- } \\
\text { tation } \\
\text { des } \\
\text { taux de } \\
\text { natalité }\end{array}$ & $\begin{array}{l}\text { Diminu- } \\
\text { tion des } \\
\text { taux de } \\
\text { natalité }\end{array}$ & $\begin{array}{l}\text { Accrois- } \\
\text { sement } \\
\text { de la } \\
\text { vieillesse } \\
\text { relative }\end{array}$ & $\begin{array}{l}\text { Acadie } \\
1755 \\
\text { Exode } \\
\text { ayant } \\
\text { un carac- } \\
\text { tère de } \\
\text { con- } \\
\text { trainte }\end{array}$ & $\begin{array}{l}\text { Révoca- } \\
\text { tion de } \\
\text { l'Edit de } \\
\text { Nantes. } \\
\text { Exode } \\
\text { des Pro- } \\
\text { testants }\end{array}$ & $\begin{array}{l}\text { Effets de } \\
\text { l'œuvre } \\
\text { de l'inten- } \\
\text { dant } \\
\text { Talon } \\
\text { en } \\
\text { Nouvelle- } \\
\text { France }\end{array}$ \\
\hline
\end{tabular}

TABLEAU II

Interprétation historique de quelques données géographiques en fonction des variables influençantes, influencées et des résultantes.

\begin{tabular}{|c|c|c|c|c|c|c|c|c|}
\hline Variables & \multicolumn{3}{|c|}{ Zones climatiques } & Transports & \multicolumn{2}{|c|}{ Relief } & \multicolumn{2}{|c|}{ Industrie } \\
\hline $\begin{array}{r}\begin{array}{r}\text { Influen- } \\
\text { chantes } \\
\text { encées }\end{array} \\
\text { cnflu- }\end{array}$ & Froid & $\begin{array}{l}\text { Tem- } \\
\text { péré }\end{array}$ & $\begin{array}{c}\text { Sous- } \\
\text { tropical }\end{array}$ & $\begin{array}{c}\text { Carrefour } \\
\text { des voies } \\
\text { de commu- } \\
\text { nication }\end{array}$ & $\begin{array}{l}\text { Région } \\
\text { des } \\
\text { vallées } \\
\text { et des } \\
\text { plaines }\end{array}$ & $\begin{array}{l}\text { Région } \\
\text { des } \\
\text { monta- } \\
\text { gnes }\end{array}$ & $\begin{array}{c}\text { Exploita- } \\
\text { tion des } \\
\text { richesses } \\
\text { natu- } \\
\text { relles } \\
\end{array}$ & $\begin{array}{l}\text { Industrie } \\
\text { de trans- } \\
\text { formation }\end{array}$ \\
\hline $\begin{array}{l}\text { Densité } \\
\text { de peu- } \\
\text { plement }\end{array}$ & Faible & Accrue & Accrue & $\begin{array}{l}\text { Accrue } \\
\text { Ex.: peuple- } \\
\text { ment amérin- } \\
\text { dien situé } \\
\text { aux futurs } \\
\text { lieux d'ag- } \\
\text { glomérations } \\
\text { urbaines }\end{array}$ & $\begin{array}{l}\text { Relati- } \\
\text { vement } \\
\text { élevée }\end{array}$ & Faible & Elevée & $\begin{array}{l}\text { Exode } \\
\text { rural; } \\
\text { croissance } \\
\text { urbaine }\end{array}$ \\
\hline
\end{tabular}

